\title{
Estudo do consumo de plantas medicinais na Região Centro-Norte do Estado do Rio de Janeiro: aceitação pelos profissionais de saúde e modo de uso pela população
}

Valdir Florencio da Veiga Junior

Departamento de Química, Universidade Federal do Amazonas, Av. Gal. Rodrigo Octávio Jordão Ramos, 3000, Japiim, 69077-000 Manaus-AM, Brasil

\begin{abstract}
RESUMO: A utilização de plantas com objetivos medicinais é bastante difundida em todo o mundo. No Brasil, são raras as pesquisas que avaliem o grau de utilização das plantas como medicamentos e sua inserção na cultura popular. A análise de 1.320 formulários preenchidos pela população do interior do estado do Rio de Janeiro e por profissionais da área de saúde permitiu verificar que as plantas medicinais são as principais formas de tratamento para $63 \%$ dos entrevistados, apesar da disponibilidade de medicamentos alopáticos. Foi observada a utilização de plantas como automedicação antes da consulta ao médico, concomitante com o medicamento alopático $(55,9 \%)$ e, em muitos casos, substituindo-o (52,4\%), sem o conhecimento do médico. A pesquisa mostrou ainda a falta de conhecimento dos profissionais da área de saúde sobre a cultura de utilização das plantas medicinais pela população, preferindo terapias alternativas que não fazem parte da cultura popular brasileira, como a acupuntura.
\end{abstract}

Unitermos: Fitoterapia, plantas medicinais, terapias alternativas.

\begin{abstract}
Study of the medicinal plants consumption in the Middle-North Region of the Rio de Janeiro State: acceptance by health professionals, way of use of the population". All over the world plants have been used for medicinal purposes. In Brazil, there is no statistical research showing cultural and popular aspects for using medicinal plants. Intoxication, interactions with last generation allopathic and loss of knowledge of using plants are critical aspects of selfmedication. The results obtained from 1.320 questionnaires, filled up at Rio de Janeiro State and by health professionals allowed us to check that medicinal plants are the main health treatment (for $63.0 \%$ ), in spite of the availability of allopathic. It is used before consulting a doctor and concomitant with allopathic (55.9\%), sometimes replacing it $(52.4 \%)$. The results of this research show a lack of information from health professionals to cultural utilization of medicinal plants by the population, preferring alternative therapies that are not part of the Brazilian popular culture, such as acupuncture.
\end{abstract}

Keywords: Phytotherapy, medicinal plants, alternative therapies.

\section{INTRODUÇ̃̃O}

O homem moderno pode ser compreendido e diferenciado das demais épocas por seu consumo elevado de medicamentos. As pesquisas químicas e farmacêuticas ao longo do Século XX possibilitaram o alívio para males que assolaram a humanidade por séculos, como a tuberculose, a sífilis, o câncer e a hanseníase, assim como para as endemias do mundo moderno, como a depressão, as cardiopatias e a AIDS. A grande oferta de medicamentos alopáticos, entretanto, não resolveu os problemas de saúde da maior parte da população. Segundo a Organização Mundial de Saúde, $80 \%$ da humanidade não têm acesso ao atendimento primário de saúde, por estarem muito distantes dos centros de saúde ou por não possuírem recursos para adquirir os medicamentos prescritos (Akerele, 1993).
Para essa população, as terapias alternativas são as principais formas de tratamento, e as plantas medicinais, os principais medicamentos (Mengue et al., 2001; Ritter et al., 2002; Mendonça-Filho \& Menezes, 2003; Pereira et al., 2004; Vendruscolo et al., 2005; Carlini et al., 2006; Agra et al., 2007; Biavatti et al., 2007).

Se a população dos países mais pobres utiliza as plantas medicinais por tradição e ausência de alternativas econômicas viáveis, nos países mais desenvolvidos observa-se um maior uso de fitomedicamentos influenciado pelos modismos de consumo de produtos naturais. Este modismo favoreceu a difusão das promessas de cura através das plantas medicinais para males como a impotência, a ansiedade e a obesidade, algumas vezes em um único extrato. $\mathrm{O}$ conceito mais perigoso surgido por esta época foi o de que as plantas medicinais não representam quaisquer riscos para a 
saúde humana por serem naturais e terem sido testadas através de séculos de utilização pela população de todo o mundo (Veiga Jr et al., 2005).

A falta de informações adequadas sobre as propriedades das plantas medicinais (principalmente das exóticas), seu consumo concomitante com os medicamentos tradicionais (alopáticos) sem aviso ao médico e, finalmente, a perda do conhecimento sobre os efeitos medicinais e tóxicos das plantas, assim como a capacidade de identificá-las pela migração da população rural para as cidades são fatores preocupantes da automedicação (Albuquerque \& Hanazaki 2006; Veiga Jr. Et al., 2005).

Na Alemanha, país que consome metade dos extratos vegetais comercializados em toda a Europa, a automedicação com plantas é a terapia alternativa mais comum da população, apesar de $70 \%$ dos médicos em clínica geral prescreverem as centenas de ervas registradas e uma parte significante destas ser paga pelo seguro de saúde (Blumentahl, 1998).

A automedicação é particularmente preocupante quando é realizada em conjunto com outros medicamentos, podendo levar a efeitos sinérgicos e interações não esperadas pelo médico. Diversos estudos vêm sendo realizados analisando a potencialização dos efeitos de diuréticos pelo dente-de-leão (Taraxacum officinale), da atividade de anti-depressivos pela ervade-São-João (Hypericum perforatum), dos efeitos de hipnóticos e ansiolíticos pelo maracujá (Passiflora incarnata) e, ainda, interações não tão óbvias, como a dos extratos à base de alho (Allium sativum) com os medicamentos que compõem o coquetel anti-HIV, como o saquinavir e o indinavir (Newall et al., 1996; Piscitelli, 2002; Cordeiro et al., 2005; Alexandre et al., 2008).

A refratariedade de grande parte da comunidade da área de saúde à utilização das plantas medicinais como opção de tratamento completa o quadro atual, de automedicação indiscriminada em todas as classes sociais, muitas vezes sem o conhecimento da toxicidade dos extratos vegetais, o que pode ser perigoso se não houver a garantia de que as propriedades farmacológicas esperadas sejam realmente obtidas, sem efeitos colaterais ou adversos perigosos para sua saúde.

A utilização de plantas associada aos rituais religiosos nas crenças populares e a ausência de testes clínicos em humanos que comprovassem as atividades farmacológicas da maioria dos extratos vegetais foram alguns dos principais motivos que levaram ao ceticismo da comunidade médica quanto a prescrição dos fitomedicamentos. Somente os medicamentos alopáticos possuíam estudos de farmacocinética, farmacodinâmica e toxicologia e poderiam ser receitados com segurança para os pacientes e médicos.

Atualmente, muitas plantas medicinais utilizadas em rituais religiosos possuem estudos científicos que comprovam suas propriedades farmacológicas e dão sentido às utilizações ritualísticas.
A arruda (Ruta graveolens), por exemplo, é empregada em banhos rituais de "proteção em casos de amor" (Farelli, 2000). Estudos recentes mostraram que um de seus constituintes, a metilnonilcetona, excita a motilidade uterina, apresentando atividade abortiva. A erva-cidreira (Melissa officinalis), utilizada em banhos de descarrego e alívio, apresenta efeitos analgésicos e tranqüilizantes, além de diminuição da insônia e da ansiedade. O mulungu (Erythrina verna), utilizado em rituais fúnebres, apresenta comprovados efeitos sedativos, podendo causar entorpecimento e bradicardia (Camargo, 1998; Corrêa et al., 1998).

Quais extratos são realmente ativos, como e quando podem ser prescritos e como devem ser preparados são questões que misturam o conhecimento popular ao científico e que devem ser respondidas através das comprovações científicas e da ampliação dos estudos das plantas brasileiras, principalmente nas áreas de fitoquímica e etnofarmacologia (Maciel et al, 2002). Somente com estas respostas, que já existem para dezenas de fitomedicamentos, e sua divulgação, os médicos passarão a prescrevê-los, aproximando-se dos desejos da população, de utilizar as plantas medicinais em seus tratamentos.

No Brasil, diretrizes do Ministério da Saúde determinaram prioridades na investigação das plantas medicinais e implantando a fitoterapia como prática oficial da medicina, orientando as Comissões Interinstituicionais de Saúde (CIS) a buscarem sua inclusão no Sistema Único de Saúde (SUS). Para que essa inclusão ocorra é essencial que os profissionais da área de saúde conheçam as atividades farmacológicas e a toxicidade das plantas medicinais de cada bioma brasileiro, de acordo com os costumes, tradições e condição sócio-econômica da população. Alguns trabalhos já são realizados em estados como o Ceará com o objetivo de desvendar o uso de plantas medicinais pela população, encontrando uma alta prevalência de uso (Silva et al, 2006).

O objetivo deste trabalho é descrever um levantamento estatístico realizado no interior do Estado do Rio de Janeiro sobre o uso e os costumes de utilização de plantas medicinais pela população e sua aceitação e conhecimento pelos profissionais de área da saúde. Não se trata de uma análise etnobotânica, com todos os cuidados que exige um estudo desta natureza (Albuquerque \& Hanazaki 2006), mas um relato do uso das plantas por uma determinada população. Por esse motivo, optou-se por omitir os nomes das plantas citadas, de forma a resguardar o conhecimento tradicional, sendo citadas somente duas plantas, de amplo uso.

\section{MATERIAL E MÉTODOS}

O levantamento estatístico foi realizado entre março e maio de 2004, na Região Serrana e Centro-Norte do Estado Rio de Janeiro, através de entrevistas com 
populares dos municípios de Cantagalo (18\%), Duas Barras (5\%), Cordeiro (28\%), Bom Jardim (9\%) e Nova Friburgo (41\%), sendo entrevistados homens (34\%) e mulheres (66\%), na zona urbana (37\%) e nos distritos da árearural $(63 \%)$, destes municípios. Paralelamente, foram entrevistados profissionais da área de saúde atuando na mesma região, incluindo os municípios de Sumidouro, Macuco, Carmo e Trajano de Morais. No total, foram preenchidos 1.100 questionários entre a população em geral, com 998 destes tendo sido considerados válidos, e outros 220 questionários preenchidos entre médicos $(10 \%)$, profissionais da área de enfermagem $(60 \%)$, fisioterapeutas $(9 \%)$, dentistas $(6 \%)$, farmacêuticos $(3 \%)$ e psicólogos $(2 \%)$, entre outros profissionais de saúde atuando na mesma região. As entrevistas foram realizadas por acadêmicos do curso de enfermagem da Universidade Estácio de Sá, Campus Nova Friburgo. A análise estatística foi realizada utilizando o método Quiquadrado, com significância fornecida como valores de $\mathrm{p}$.

\section{RESULTADOS E DISCUSSÃO}

Ao contrário do que tem ocorrido nos países Europeus, Asiáticos e nos Estados Unidos, o Brasil praticamente não dispõe de estatísticas que expliquem o mercado, o consumo e os costumes de uso de plantas medicinais, apesar de existir grande tradição de seu uso em vários biomas, como a Amazônia, o Cerrado e a Mata Atlântica. Nos poucos estudos etnofarmacológicos produzidos no Brasil, a incidência dos relatos de uso de plantas exóticas é imensa, em detrimento às plantas nativas.

A região serrana do Rio de Janeiro, nas proximidades de Nova Friburgo, é um dos últimos refúgios de Mata Atlântica protegido por reservas biológicas, como a de Três Picos (com 46.350 ha), com centros urbanos bem desenvolvidos (Nova Friburgo possui mais de 177.000 habitantes) e municípios com grande parte da população essencialmente rural (IBGE, 2007).

No primeiro item da pesquisa, realizada com pequena amostra de populares da área urbana de Nova Friburgo, os entrevistados foram perguntados se faziam uso de plantas medicinais. Como resultado inicial, que motivou a ampliação da pesquisa, foi observado que um elevado percentual da população, acima dos $90 \%$, fazia uso regularmente de plantas para a cura de seus males. Com a finalização da pesquisa foi observado que $97,7 \%$ dos entrevistados afirmaram utilizar plantas para fins medicinais regularmente e apenas 2,3\% disseram não utilizá-las no dia-a-dia. Mais de dois terços dos entrevistados $(67,9 \%)$ afirmaram estar fazendo uso de alguma planta medicinal durante o período em que a pesquisa foi realizada. Em pesquisa semelhante, realizada nos Estados Unidos, em 1997, observouse que $42 \%$ da população haviam feito uso de plantas medicinais pelo menos uma vez no ano de 1996, em tratamentos médicos alternativos (Eisenberg, 1998), o que foi considerado um percentual elevado.

Para cerca de $63,0 \%$ dos entrevistados, as plantas medicinais são utilizadas sempre que há algum tipo de indisposição ou problema de saúde, sendo que apenas $12,6 \%$ destes vêem a utilização das plantas medicinais com alguma ressalva, preferindo utilizálas somente nos casos mais simples, como gripes e pequenas infecções. A utilização somente nos casos terminais aparece como uma alternativa para $1,4 \%$ dos entrevistados na pesquisa.

Entre as plantas mais freqüentemente utilizadas, foram citados o boldo brasileiro (Plectanthus barbatus) e a camomila (Chamomila recutita), em $14,7 \%$ e $7,0 \%$ dos formulários, respectivamente.

\section{A difusão do conhecimento das plantas medicinais}

A urbanização das cidades e a migração da população rural para a área urbana levam à perda do conhecimento sobre as plantas medicinais. Seja em função do distanciamento das plantas (nas áreas urbanas os quintais com jardins, onde as plantas possam ser reconhecidas e coletadas, são cada vez menos freqüentes) ou da falta de interesse no aprendizado de suas propriedades, as novas gerações parecem estar perdendo este conhecimento, acumulado pelos seus antepassados.

Quando perguntados sobre quem prepara as plantas medicinais na família, $44,5 \%$ dos pesquisados responderam que são as mulheres, cabendo a apenas $5,7 \%$ dos homens a mesma tarefa. Para $28,4 \%$ dos entrevistados o conhecimento sobre a preparação dos extratos à base de plantas é bem difundido em toda família, enquanto em $21,4 \%$ dos casos, entretanto, somente os mais velhos detêm este conhecimento, como ilustra a Figura 1.

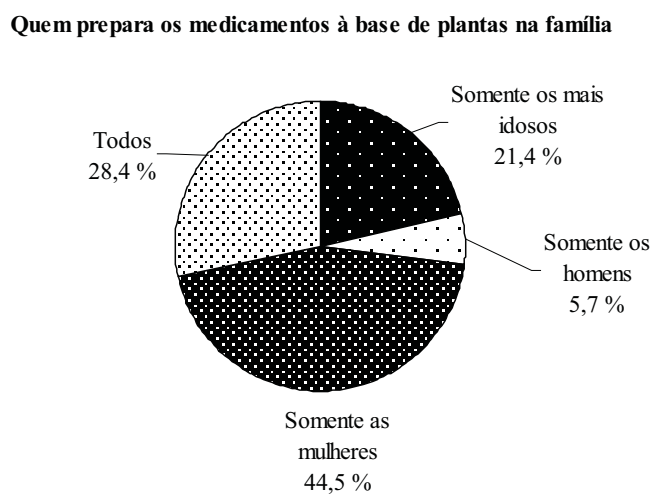

Figura 1. Preparo de plantas medicinais na família

O baixo percentual de homens que realizam as preparações $(5,7 \%)$ está relacionado, em geral, com a facilidade de obtenção das plantas medicinais. Quando as plantas utilizadas são espécies nativas selvagens a sua 
busca se dá no interior da mata e os homens da família são geralmente os escolhidos para sua coleta e, geralmente, também preparam os extratos vegetais, situação comum na Amazônia, por exemplo. Este resultado evidencia a utilização de plantas comercializadas ou domesticadas.

A preparação das formulações medicinais à base de plantas é realizada apenas pelos mais idosos para $21,4 \%$ dos entrevistados, porcentagem pouco menor que os $28,4 \%$ dos casos em que foi relatada a preparação por todos na família. O alto percentual de mulheres que preparam as formulações $(44,5 \%)$ é condizente com o conhecimento obtido em estudos etnofarmacológicos anteriores de que são as mulheres, que normalmente preparam os alimentos, que também preparam os chás e as infusões à base de plantas (Howard, 2003).

Quando perguntados sobre quem indica as plantas a serem utilizadas para cada doença, cerca de 90,1\% dos entrevistados responderam ter aprendido com a família ou pessoas próximas, como amigos e parentes. De todos os entrevistados, somente 3,1\% responderam utilizar as plantas medicinais com indicação médica e $1,1 \%$ com indicação de agentes de saúde. Somandose os dois resultados observa-se que apenas $4,2 \%$ têm indicação de profissionais da área de saúde, porcentagem muito pequena, devendo, no contexto já apresentado de disseminação do uso dos medicamentos à base de plantas medicinais pelo SUS, ser elevada através da formação especializada dos profissionais de saúde. A indicação da planta é proveniente de mateiros e rezadeiras para $5,4 \%$ dos entrevistados, percentual maior do que aquele obtido para os profissionais graduados da área de saúde. A atuação de padres e pastores foi de apenas $0,4 \%$, porcentagem bastante diferente da encontrada no passado, quando as pastorais apresentavam atuação marcante na disseminação da medicina popular e caseira (Camargo, 1998; Franco \& Fontana, 2003).

Para $76,1 \%$ dos entrevistados, as plantas medicinais que utilizam são obtidas na própria casa $(39,9 \%)$, da família, de conhecidos ou na rua. A aquisição da planta medicinal só é realizada com maior preocupação com a qualidade para pouco mais de $16 \%$ dos entrevistados, que as adquirem em lojas específicas de produtos naturais $(10,9 \%)$, farmácias $(1,6 \%)$ e farmácias de manipulação $(3,6 \%)$. Mateiros e rezadeiras fornecem para $4,5 \%$ dos entrevistados. Agente de saúde $(1,7 \%)$, padres e pastores $(1,5 \%)$ são, juntos, responsáveis por pouco mais de $3 \%$ das indicações. O elevado percentual de quase $40 \%$ dos entrevistados que utilizam plantas de seu próprio jardim é condizente com o esperado para a região e traduz um grande conhecimento e proximidade desta população com as plantas.

$\mathrm{O}$ risco, neste caso, seria o da identificação errônea da planta. Um aumento deste percentual com garantias da qualidade do produto utilizado poderia ser alcançado com a adoção das chamadas "farmáciasvivas". Através delas, hortas de plantas medicinais poderiam ser instaladas próximas aos postos de saúde e

centros comunitários fornecendo as plantas medicinais gratuitamente, para a população mais carente, que poderia compor sua farmácia caseira com plantas certificadas por botânicos e ainda serem instruídos das melhores práticas de sua utilização, com a formulação e a dosagem adequados, orientados por um farmacêutico responsável (Matos, 1998).

\section{Riscos da automedicação}

A forma de utilização é importante não somente para a garantia de presença do princípio ativo, mas, também, para a certificação de baixa toxicidade (Eldin \& Dunford, 2001). A utilização das plantas medicinais por meio de chás e infusões é citada por $60,2 \%$ dos entrevistados. Somados aos 18,9\%, que fazem uso de garrafadas (extratos alcoólicos ou xaropes obtidos de várias plantas), obtém-se um percentual próximo a $80 \%$ dos entrevistados que ingerem os preparados à base de plantas medicinais. A utilização das plantas medicinais e seus extratos em cápsulas, obtidas em farmácias, preparadas com princípios de maior qualidade e higiene, é citada por apenas 2,4\% dos entrevistados. Quando se faz uso de extratos à base de plantas medicinais, obtidos de fonte não segura, através de ingestão, são grandes os riscos de intoxicação. Nesse caso, mais de $82 \%$ dos entrevistados optam por esta forma de administração.

As dermatites, efeito adverso mais comum das plantas medicinais, ocorrem principalmente quando a planta é utilizada através de emplastos ou em aplicação tópica. Esta forma de utilização foi observada em 12,1\% das citações.

Outra forma de utilização das plantas medicinais é através dos chamados alimentos funcionais, com o consumo de vegetais ricos em ferro, em casos de anemia, ou alimentos ricos em vitamina $\mathrm{C}$, quando há uma necessidade de fortalecimento do sistema imunológico, por exemplo. A utilização das plantas medicinais na alimentação corrente é uma forma de tratamento citada por apenas $6,4 \%$ dos entrevistados.

A automedicação utilizando as plantas medicinais é um procedimento particularmente perigoso quando é realizado substituindo o medicamento alopático. Apenas 34,4\% atestaram não praticá-la. Para $13,2 \%$ dos entrevistados a substituição ocorre quando o medicamento alopático é mais caro ou julgam não resolver seu problema, procedimento bastante perigoso uma vez que determinados tratamentos, como o combate à tuberculose, envolvem vários meses de medicação ininterrupta, a maior parte deles após o desaparecimento dos sintomas.

Para a maioria dos entrevistados $(52,4 \%)$ a planta medicinal sempre (ou sempre que se conhece uma planta que possa ser empregada) é utilizada para substituir o medicamento de farmácia. Analisando este resultado mais profundamente observam-se diferenças significativas $(\mathrm{p} \leq 0,01)$ em relação à faixa 
etária e à região de moradia (rural ou urbana) entre os indivíduos que substituem os medicamentos alopáticos receitados pelos médicos por plantas medicinais, mas não em relação ao sexo. A diferença de acordo com a região de origem é a mais marcante. Entre a população rural, $73,1 \%$ dos entrevistados relataram substituir o medicamento alopático pelas plantas, contra somente $57,2 \%$, entre a população urbana pesquisada. Entre os indivíduos que possuem entre 50 e 69 anos, 75,9\% responderam substituir os medicamentos das farmácias pelas plantas medicinais, enquanto que na faixa etária de 18 a 30 anos esta substituição ocorre para apenas $53,9 \%$ dos entrevistados.

Quando perguntados se substituiriam o medicamento alopático pela planta medicinal se houvesse indicação médica, $65,1 \%$ disseram que sim. Somente $12,2 \%$ disseram que não utilizariam, demonstrando preferência pelo medicamento alopático, quando receitado pelo médico. Para $17,8 \%$ dos entrevistados, os medicamentos à base de plantas medicinais só seriam utilizados para o tratamento dos casos mais simples, como resfriados, enquanto $2,7 \%$ mostraram preocupação com a garantia de qualidade, atestando que só substituiriam o alopático pela planta medicinal se esta fosse vendida na farmácia.

Estes resultados confirmam a confiança da população no médico e também no efeito das plantas medicinais, demonstrando que uma conjugação da medicina tradicional com a popular seria bem aceita pela população, da mesma forma que já ocorre em países como a China e a Índia.

\section{Interações medicamentosas}

A atitude que pode elevar a incidência de efeitos adversos provocados pela associação das plantas medicinais com os medicamentos alopáticos também foi avaliada. Quando perguntados se utilizam formulações contendo plantas medicinais em conjunto com os medicamentos alopáticos, $44,1 \%$ relataram não utilizar (Figura 2). Outros $8,4 \%$ relatam que utilizam plantas medicinais em conjunto com o medicamento alopático quando o alopático é muito caro ou quando crêem que não funciona. $\mathrm{O}$ número mais importante deste item da pesquisa é que, para $47,5 \%$ dos entrevistados, as plantas medicinais são utilizadas em conjunto com o medicamento de farmácia sempre que conhece uma planta que possa ser indicada.

Se as práticas de automedicação, substituição dos alopáticos pelas plantas e uso concomitante representam riscos para a população, o perigo é ainda maior quando o médico não é informado deste procedimento. Quando perguntados se notificam ao médico quando utilizam o medicamento alopático receitado em conjunto com as plantas medicinais, $59,4 \%$ relatam que nunca avisam ou somente se passarem mal, enquanto somente $40,6 \%$

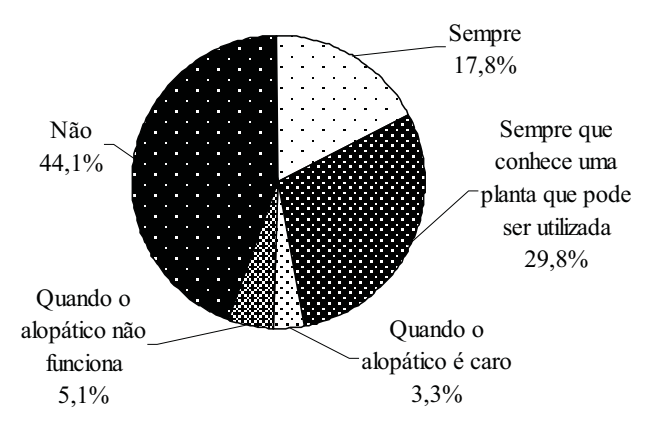

Figura 2. Utilização das plantas medicinais em conjunto com os medicamentos alopáticos

disseram sempre (ou ocasionalmente) relatar ao médico o uso concomitante de plantas e alopáticos.

\section{A visão dos profissionais de saúde}

No universo de 220 profissionais de saúde pesquisado, $65 \%$ foram do sexo feminino e $35 \%$ do sexo masculino, atuando em instituições privadas (31\%), públicas (50\%) e como autônomos (19\%).

Quando perguntados qual a terapia alternativa mais prescrita, os profissionais de saúde responderam que a acupuntura é a técnica mais aconselhada, indicada em $45 \%$ dos questionários. A fitoterapia, em segundo lugar, recebeu a indicação de $27 \%$ dos profissionais, próxima dos $20 \%$ que indicam a homeopatia. Terapias ortomoleculares, shiatsu e ioga também foram citadas.

De forma geral, $27 \%$ dos profissionais entrevistados julgam que as terapias alternativas não sejam seguras por provocarem alergias (17), problemas de visão (16), náuseas (11), vômitos (10) e distúrbios hormonais (7). Por esta razão $41 \%$ dos profissionais não indicam as terapias alternativas.

Com relação à automedicação, os profissionais da área de saúde que participaram desta pesquisa julgaram que $43 \%$ da população procuram o médico já automedicados, que $25 \%$ não utilizam plantas medicinais e que apenas $32 \%$ as utilizam regularmente.

\section{CONCLUSÃO}

O conjunto de resultados obtidos nesta pesquisa nos permite visualizar grandes riscos de saúde para a população que se automedica, mas, por outro lado, sinaliza para um mercado potencialmente promissor, em relação à atuação dos profissionais da área de saúde na área de plantas medicinais.

A prevalência de utilização de plantas medicinais foi muito elevada, alcançando $97,7 \%$ do total de entrevistados. A automedicação aparece como um dos principais riscos para a população. A perda potencial do conhecimento sobre a identificação e a função das plantas medicinais (para 21,4\% dos entrevistados 
somente as pessoas mais idosas detêm o conhecimento das plantas medicinais), a aquisição da planta de fontes não certificadas e a substituição do medicamento alopático pelos extratos de plantas medicinais, ou seu uso concomitante sem notificação ao médico, trazem grandes riscos de aparecimento de efeitos adversos e intoxicações, além de alterar os resultados esperados para os medicamentos alopáticos. Os profissionais da área de saúde, por sua vez, demonstram grande reticência na indicação das plantas medicinais, especialmente pelo receio dos efeitos adversos. Por esta razão, indicam a acupuntura como principal terapia alternativa, e não a fitoterapia, que é amplamente utilizada para a população.

Por outro lado, a maior parte dos entrevistados declarou aprovar o uso das plantas medicinais com indicação médica, o que explicita a aceitação da população para a utilização de extratos comercializados e aponta para a solução do problema formulado acima: a maior especialização dos profissionais da área de saúde na aplicação e indicação das plantas medicinais.

\section{REFERÊNCIAS}

Agra MF, França PF, Barbosa-Filho JM 2007. Synopsis of the plants known as medicinal and poisonous in Northeast of Brazil. Rev Bras Farmacogn 17: 114-140.

Akerele O 1993. Summary of WHO guidelines for assessment of herbal medicines. HerbalGram 28: 13-19.

Albuquerque UP, Hanazaki N 2006. As pesquisas etnodirigidas na descoberta de novos fármacos de interesse médico e farmacêutico: fragilidades e perspectivas. Rev Bras Farmacogn 16 (Supl): 678-689.

Alexandre RF, Bagatini F, Simões CMO 2008. Interações entre fármacos e medicamentos fitoterápicos à base de ginkgo ou ginseng. Rev Bras Farmacogn 18: 117-126.

Biavatti MW, Marensi V, Leite SN, Reis A 2007. Ethnopharmacognostic survey on botanical compendia for potential cosmeceutic species from Atlantic Forest. Rev Bras Farmacogn 17: 640-653

Blumentahl M 1998. The Complete German Commission E Monographs: Therapeutic Guide to Herbal Medicines. Austin: American Botanical Council.

Camargo MTLA 1998. Plantas Medicinais e de Rituais Afrobrasileiros II, São Paulo: Ícone editora.

Carlini EA, Rodrigues E, Mendes FR, Tabach R, Gianfratti B 2006. Treatment of drug dependence with Brazilian herbal medicines. Rev Bras Farmacogn 16: 690-695.

Cordeiro CHG, Chung MC, Sacramento LVS 2005. Interações medicamentosas de fitoterápicos e fármacos: Hypericum perforatum e Piper methysticum. Rev Bras Farmacogn 15: 272-278.

Corrêa AD, Siqueira-Batista R, Quintas LE 1998. Plantas Medicinais do Cultivo à terapêtica. Petrópolis: Ed. Vozes.

Eisenberg D 1998. Trends in alternative medicine use in the United States; 1990-1997. J Am Med Assoc 280: 1569-1575.
Eldin S, Dunford A 2001. Fitoterapia na atenção primária à saúde, Barueri: Editora Manole.

Farelli MH 2000. Plantas que curam e cortam feitiços. $6^{\mathrm{a}}$. Edição. Rio de Janeiro: Editora Pallas.

Franco IJ, Fontana, VL 2003. Ervas e Plantas - A Medicina dos Simples. ${ }^{\text {a }}$. Edição, Erexim, Editora Edelbra.

Howard PL 2003. Women \& Plants. Gender Relations in Biodiversity Management \& Conservation. Londres: Zed Books Ltd.

IBGE 2007. Fundação Instituto Brasileiro de Geografia e Estatística. Estatísticas das cidades 2007. http:// www.ibge.gov.br (acessado em 29/Fev/2008).

Maciel MAM, Pinto AC, Veiga Jr. VF, Echevarria A, Grynberg NF 2002. Plantas Medicinais: a necessidade de estudos multidisciplinares. Quim Nova 25: 429-438.

Matos FJA 1998. Farmácias Vivas, 3 ${ }^{\mathrm{a}}$. Edição, Fortaleza: Editora UFC.

Mendonça-Filho RFW, Menezes FS 2003. Estudo da utilização de plantas medicinais pela população da Ilha GrandeRJ. Rev Bras Farmacogn 13(Supl): 55-58.

Mengue SS, Mentz LA, Schenkel EP 2001. Uso de plantas medicinais na gravidez. Rev Bras Farmacogn 11: 21-35.

Newall CA, Anderson LA, Phillipson JD 1996. Herbal Medicines: A guide for health-care professionals. London: The Pharmaceutical Press.

Pereira RC, Oliveira MTR, Lemos GCS 2004. Plantas utilizadas como medicinais no município de Campos de Goytacazes - RJ. Rev Bras Farmacogn 14 (Supl. 1): $37-40$.

Piscitelli SC 2002. The effect of garlic supplements on the pharmacokinetics of saquinavir. Clin Infect Dis 34: 234-238.

Ritter MR, Sobierajski GR, Schenkel EP, Menth LA. Plantas usadas como medicinais no município de Ipê, RS, Brasil. Rev Bras Farmacogn 12: 51-62.

Silva MIG, Gondim APS, Nunes IFS, Sousa FCF 2006. Utilização de fitoterápicos nas unidades básicas de atenção à saúde da família no município de Maracanaú (CE). Rev Bras Farmacogn 16: 455-462.

Veiga Jr. VF, Maciel MAM, Pinto AC 2005. Plantas medicinais: cura segura? Quim Nova 28: 519-528.

Vendruscolo GS, Rates SMK, Mentz LA 2005. Dados químicos e farmacológicos sobre as plantas utilizadas como medicinais pela comunidade do bairro Ponta Grossa, Porto Alegre, Rio Grande do Sul. Rev Bras Farmacogn 15: 361-372. 